\title{
The Cooling Simulation of Microwave Chip Based on I-DEAS ESC
}

\author{
Bin Ye \\ Shandong vocational college of light industry, Shandong, Zbo, 255300, China.
}

Keywords: I-DEAS ESC; Model; Microwave cooling; Simulation

\begin{abstract}
This paper mainly studies the cooling of microwave chip, based on ESC I-DEAS. Firstly, the ESC thermal analysis theory is studied, and then the finite element model of microwave chip is set up. In the third part, the calculation results are analyzed. Finally, the simulation studies are summed up.
\end{abstract}

\section{Introduction}

I-DEAS software is the product of Structural Dynamics Research Corporation. I-DEAS software is a solution which is fully integrated and used in the twenty-first Century. The complete scheme of perforative is from conception to the design development and production of the whole process, covering the mechanical design automation, product data management, collaborative products business and information engineering and implementation services etc. Simulation is one of the modules, and the Simulation Modeling Set is the finite element modeling and result visualization tool in MDA integrated environment.

\section{ESC thermal analysis theory}

ESC thermal analysis uses the lumped capacity finite difference method. This method based on local and global energy balance principle of the target is divided into plurality of control areas, each of which is used in the lumped parameter method and the equilibrium equation is set up, the heat balance equation of a control area is as follows:

$$
\int_{b} q_{n} d b+\int_{V} q_{0} d v=\int_{V} \rho c\left(\frac{\partial T}{\partial t}\right) d v
$$

In the formula:b is the control body boundary; $v$ is the volume of control volume; $q$ is the heat flow over the control bound ary ; $\mathrm{q} 0$ is the heat generation flow in control body.; $\rho$ is the density; $\mathrm{c}$ is specific heat; $\mathrm{T}$ is the temperature; $\mathrm{t}$ is time.

Formula (1) is also expressed as:

$$
\frac{\mathrm{dU}}{\mathrm{dt}}=\varrho \mathrm{eV} \frac{\mathrm{dT}_{\mathrm{i}}}{\mathrm{dt}}=\mathrm{C} \frac{\mathrm{dT}_{\mathrm{i}}}{\mathrm{dt}}
$$

Heat exchange between adjacent control volume is:

Qij $=$ Gij $\times f(T i, T j)$

In the formula:

Gij is the control volume at the adjacent heat exchange coefficient; $\mathrm{Ti}, \mathrm{Tj}$ are respectively $\mathrm{I}, \mathrm{J}$ is regional differential point temperature. The heat transfer coefficient of the adjacent regions is reflected in the heat transfer coefficient $\mathrm{G}$. Therefore, the establishment of boundary conditions is to determine the heat transfer coefficient of each adjacent region $\mathrm{G}$.

The heat balance equation of a control volume I can be expressed as formula (4):

$$
Q_{i}+\sum G_{i j} \times f\left(T_{i}, T_{j}\right)=C_{i} \frac{d T_{i}}{d t}
$$

The formula (3) for steady state thermal analy sis is obtained: 


$$
\mathrm{Q}_{\mathrm{i}}+\sum \mathrm{G}_{\mathrm{ij}} \times f\left(\mathrm{~T}_{\mathrm{i}}, \mathrm{T}_{\mathrm{j}}\right)=0
$$

Every geometric element as the control volume in I-DEAS ESC which then creates the finite element mesh can be directly conduct a finite difference analysis model so as to avoid the problem that a complex structure is hard to classify the finite difference grid of. The finite element method can be used to build the model, and then calculated by the finite difference method, which is the most outstanding advantages of I-DEAS software thermal analy sis.

\section{Finite element model for microwave chip heat dissipation calculation}

When a microwave generator works, the ambient temperature is as high as $50^{\circ} \mathrm{C}$. The dissipated energy of the chip (per 62.5W), only a small portion of the energy is converted into useful energy, and most of them becomes heat energy, making the intemal and heat of the instrument. The temperature range for the normal work of the electronic component is less than or equal to $175^{\circ} \mathrm{C}$. With forced cooling, fan ventilation for each $0.035 \mathrm{~m} 3 / \mathrm{s}$, a total of three, chip layout and fine position as showed in Fig. 1 .

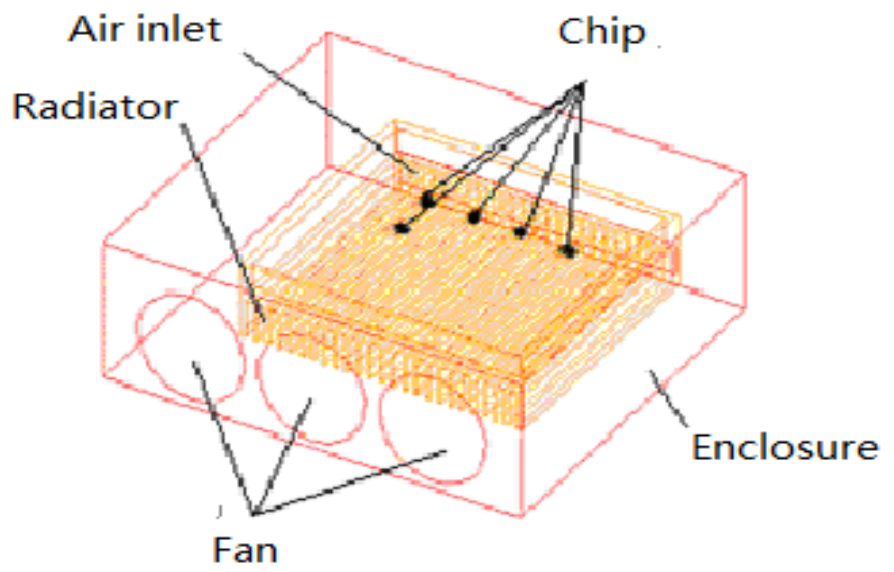

Fig. 1 Microwave case structure

Finite element mesh. Modeling is established radiator and box model. Finally, the second assembly, which heat sinks material for copper body material for air. The mesh is divided into four nodes in tetrahedral nodes. According to the structural features, the model partition is firstly divided, and then different regions are divided by different unit length.

Because the chip is small compared with the chassis, the distortion and error caused by the difference of mesh size is avoided, and the radiator is modeled with the actual size of the solid model, and the solid element is used. The chip is replaced with the surface of the radiator correspond ing to the radiator, and the chip is used for the shell element. Heat conduction coupling is between the radiator unit and the chip unit.The finite element model consists of 18642 units and 4579 nodes.

Boundary conditions. The reasonable simplification and loading of boundary conditions determined the reliability and accuracy of thermal analysis, and the actual cooling condition of the chassis, and the boundary con ditions of the model are considered to be follows.

1) Chip

Chip is the heat source of the microwave generator. The heat source is in charge on the surface of the radiator in a concentrated heat load mode, and the total power of the chip is numerically.

2) Internal air and radiator surface of the case

By using the method of partition, the air area is divided into a solid unit of large size, and the air and heat sink is treated as internal heat conduction by ESC Blockeg.

3) Fan and inlet

Fans do not need to establish the solid model. As long as establish the air inlet area with the diameter of the fan in the chassis surface corresponding position, use theSURFACE SPLIT co mmand 
and diameter can be divided into shell unit. Load fan parameters in the area. Fan parameters in advance with fan-curve definition is included into the wind, blowing or suction (this example for ventilation, air intake, fan power.

4) Inlet and outlet settings

In the forced cooling fan inlet and outlet tuyere layout should avoid short circuit of air, in size and position should make the air in the chassis formed the greatest degree of disturbed flow to improve the heat dissipation effect. The air outlet is set with VENT command, and the size and the best position of the outlet can be determined by calculation.

5) Case and environment

The heat transfer between the case and the ambient air is natural convection, and the case is determined by the specified temperature and gravity acceleration direction.

According to the worst thermal conditions, the total energy of the chip is changed to heat energy, and the temperature of the environment is 50 .

\section{Calculation results and analysis}

ESC I-DEAS can display the results of different parts according to the need, the temperature of the chip in this case is higher, and the temperature distribution draw the most attention. When the temperature of the environment is 50, Fig. 2 is the temperature field of the chip location, and Fig. 3 is the air flow vector diagramin the case.

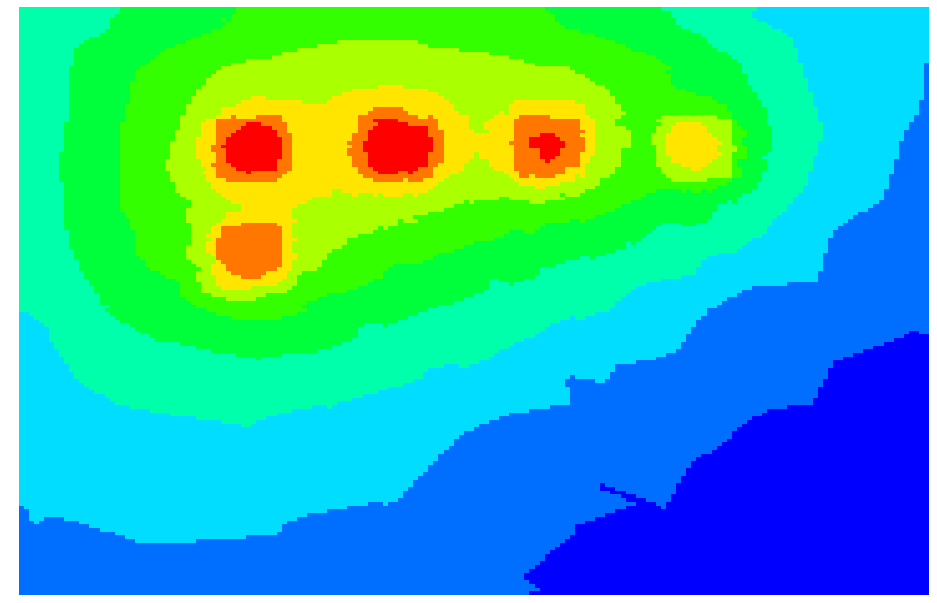

Fig. 2 Chip position temperature distribution



Fig. 3 Air flow chart in the case 
According to the calculation results, intermediate chip thermal condition is relatively poor, the highest temperature in the chip which is 167.6 degrees Celsius. Due to the adoption of the copper radiator, temperature difference between the various chips is smaller. The maximu m temperature 0.6 degrees Celsius is ideal. When the temperature is less than 175 Celsius degree, the chip can work normally.

The experimental tests are carried out and 10 temperatures measuring points were set up. Calculating points corresponding to the position of the points in the calculation model are extracted. The results of the two are shown in Table 1.

\begin{tabular}{|c|c|c|c|c|c|}
\hline \multirow[b]{2}{*}{$\begin{array}{l}\text { measurin } \\
\text { g point }\end{array}$} & \multirow{2}{*}{$\begin{array}{l}\quad \text { computat } \\
\text { ional model } \\
\text { node } \\
\text { number }\end{array}$} & \multirow{2}{*}{$\begin{array}{l}\quad \text { cal } \\
\text { cul-ati } \\
\text { on } \\
\text { value } \\
\left({ }^{\circ} \mathrm{C}\right)\end{array}$} & \multirow{2}{*}{$\begin{array}{l}\quad \text { me } \\
\text { asur-e } \\
\mathrm{d} \\
\text { value } \\
\left({ }^{\circ} \mathrm{C}\right)\end{array}$} & \multicolumn{2}{|c|}{ Error } \\
\hline & & & & $\mathrm{T}^{\Delta}$ & $(\%)$ \\
\hline 1 & 20487 & 166.7 & 165 & 1.7 & 1.03 \\
\hline 2 & 20483 & 168.9 & 170 & -1.1 & -0.65 \\
\hline 3 & 20451 & 168.9 & 170 & -1.1 & -0.65 \\
\hline 4 & 26800 & 166.8 & 164 & 2.8 & 1.7 \\
\hline 5 & 36484 & 170.7 & 168 & 2.7 & 1.6 \\
\hline 6 & 37191 & 165.7 & 164 & 1.7 & 1.03 \\
\hline 7 & 37093 & 164.7 & 164 & 0.7 & 0.43 \\
\hline 8 & 36545 & 154.6 & 155 & -0.4 & -0.26 \\
\hline 9 & 36903 & 155.1 & 156 & -0.9 & -0.58 \\
\hline 10 & 36850 & 154.5 & 153 & 1.5 & 0.98 \\
\hline
\end{tabular}

Table 1 The results were compared with the measured values

\section{Conclusions}

The arrival of the Internet era has brought an immeasurable effect to the development of CAE technology. Now, many large software companies have used the Intemet to provide technical support for the difficulties encountered during the analysis of users. With the continuous development of Intemet technology and the popularity of, passing through the information network, not only for some technical problems, even for comprehensive analysis of CAE have likely to get expert technical support, which will play a very important role in the popularization and application of CAE technology. I-DEAS ESC is an effective and practical cooling numerical simulation method for electronic systems, which can greatly reduce the computation scale and increase accuracy. The modeling method used in this paper can be widely used in thermal analysis of various electronic devices.

\section{References}

[1] Hongwu Zhang, Zhenqun Guan, Yunpeng Li, Yunxian Gu. Finite element analysis and CAE based [M]. Beijing: Tsing hua University press, 2004.

[2] Hong Ye. I-DEAS thermal analysis practical tutorial [M]. China University of technology and Technology Press, 2003.

[3] Ridong Liao. I-DEAS examples: finite element analysis [M]. Beijing Institute of Technology presses, 2003.

[4] Deren Xie. Thermal design of electronic equipment [M]. Southeast University press, 1989:189-194. 
[5] Baoyu Sun, Jun Xue. Application of thermal analysis in thermal design of electronic equipment [J]. Chang chun University of Technology press, 2007, (2), 176-179.

[6] Wenbin Huang, Ren Wang. Plastic mechanics theory [M]. Peking University press, 1982.

[7] Lu Zhou, Fuqun Fan. Mechanics of composite materials [M]. Beijing higher education press, 1991.

[8] Youliang Jiang. Nonlinear finite element method [M]. Beijing Institute of industry, press, 1988.

[9] Tao Xu. Numerical calculation method [M]. Jilin science and Technology Press, 1998.7.

[10] Kezhi Huang. Nonlinear continuummechanics [M]. Tsinghua University press, 1989. 